\title{
Особенности затухания и усиления терагерцовых плазмонных мод в графене с учетом пространственной дисперсии
}

\author{
(C) О.В. Полищук ${ }^{1}$, Д.В. Фатеев ${ }^{1,2}$, В.В. Попов ${ }^{1}$ \\ ${ }^{1}$ Саратовский фрилиал Института радиотехники и электроники им. В.А. Котельникова Российской академии наук, \\ 410019 Саратов, Россия \\ ${ }^{2}$ Саратовский государственный университет, \\ 410012 Саратов, Россия \\ E-mail: polischuk.sfire@mail.ru; popov_slava@yahoo.co.uk
}

Поступила в Редакцию 12 апреля 2021 г.

В окончательной редакции 19 апреля 2021 г.

Принята к публикации 19 апреля 2021 г.

\begin{abstract}
Рассматривается влияние дрейфа носителей заряда на моды плазмонных возбуждений (плазмонов) в электронной дираковской жидкости в графене со смещенным уровнем Ферми. Дисперсионные соотношения для плазмонов получены с использованием электродинамического подхода и гидродинамического описания электронной жидкости. Численно изучены затухающие и усиливающиеся плазмонные моды в зависимости от соотношения величин и направлений постоянного электрического тока и фазовой скорости плазмона.
\end{abstract}

Ключевые слова: терагерцовый диапазон, плазмон, графен, дрейф носителей заряда.

DOI: 10.21883/FTP.2021.10.51432.29

\section{1. Введение}

Плазменные колебания терагерцового (ТГц) диапазона в графене интенсивно изучаются [1] с момента открытия графеновых материалов [2]. Интерес к графеновой плазмонике в большой степени вызван достижимостью высокой подвижности носителей заряда в графене [3] по сравнению с подвижностью электронов в квантовых ямах полупроводниковых гетероструктур [4]. Плазменные колебания (плазмоны) в графеновых структурах характеризуются очень малыми длинами волн (на 2-3 порядка величины меньшими, чем длина электромагнитной волны ТГц частотного диапазона), а также сильной локализацией ТГц поля вблизи графена [5]. Это позволяет сконцентрировать ТГц поле в очень малом объеме (с субмикрометровыми размерами) и тем самым значительно увеличить эффективность взаимодействия ТГц поля с графеном.

В ряде работ была показана возможность создания ТГц фотонных и плазмонных усилителей и источников в структурах на основе активного графена [6-8] за счет создания инверсной населенности носителей заряда в графене $[9,10]$. В качестве различных физических механизмов накачки графена были предложены: оптический [11-15], инжекционный $[8,16]$, диффузионный [17] и механизм Ландау-Зинера [18].

Плазмоны в графеновых структурах могут возбуждаться в широком диапазоне ТГц частот [19]. Таким образом, исследование плазменных колебаний в графене в качестве платформы для создания эффективных компактных устройств управления ТГц излучением является крайне актуальным. В нижней части ТГц спектра вместо кинетической модели транспорта электронов в графене может быть использовано гидродинамическое описание электронной жидкости [20], поскольку частота быстрых электрон-электронных столкновений составляет величину $\sim 5$ ТГц согласно как экспериментальным [21], так и теоретическим оценкам с использованием микроскопической теории [22].

В данной работе рассматривается влияние дрейфа носителей заряда (вследствие приложенного к графену постоянного электрического поля) на моды плазмонных возбуждений в электронной дираковской жидкости в графене со смещенным уровнем Ферми при учете пространственной дисперсии. Показана возможность усиления плазмонов в ТГц диапазоне за счет их взаимодействия с дрейфом носителей заряда в графене.

\section{2. Постановка задачи и метод решения}

Нелокальная комплексная динамическая проводимость графена может быть получена в рамках гидродинамической модели с учетом давления электронной жидкости, постоянного электрического поля в графене и адиабатического процесса распространения волн. Для этого необходимо решить уравнение непрерывности

$$
\frac{\partial N}{\partial t}+\frac{\partial(N \mathbf{V})}{\partial x}=0
$$

где $x, t-$ координата и время соответственно, $N-$ концентрация частиц, $V$ - гидродинамическая скорость носителей заряда, и уравнение баланса импульса носителей заряда

$$
\frac{\partial \mathbf{S}}{\partial t}+\nabla \hat{\Pi}+e \mathbf{E} N+\frac{e}{c} \mathbf{J} \times \mathbf{B}=-\mathbf{S} \gamma,
$$

где $e-$ заряд электрона, $c-$ скорость света, $\gamma=1 / \tau$, $\tau$ - феноменологическое время рассеяния импульса 
носителей заряда в графене, $\mathbf{S}$ - макроскопический импульс частиц (носителей заряда), $\hat{\Pi}$ - тензор плотности потока импульса, $\mathbf{E}$ и $\mathbf{B}$ - электрическое и магнитное поля соответственно, $\mathbf{J}$ - плотность постоянного тока, совместно с уравнением баланса энергии

$$
\frac{\partial W}{\partial t}+\mathbf{V}_{\mathrm{F}}^{2} \nabla \mathbf{S}-e \mathbf{E} \times \mathbf{J}=0,
$$

где $W$ - макроскопическая энергия.

Физические величины, входящие в уравнения (1)-(3), связаны между собой следующими соотношениями [23-25]:

$$
\begin{gathered}
\mathbf{S}=M \mathbf{V}, \\
\hat{\Pi}=P+\mathbf{S} \otimes \mathbf{V}, \\
P=M\left(\mathbf{V}_{\mathrm{F}}^{2}-\mathbf{V}^{2}\right) / 3, \\
W=M \mathbf{V}_{\mathrm{F}}^{2}-P,
\end{gathered}
$$

где $M$ - плотность электронной жидкости, $P$ - давление электронной жидкости, $\mathbf{V}_{\mathrm{F}}-$ скорость Ферми в графене. Отметим, что время релаксации энергии намного больше, чем период колебаний, поэтому процесс преобразования энергии является адиабатическим, что приводит к равенству нулю правой части уравнения (3).

Уравнения гидродинамики (1)-(3), совместно с соотношениями (4)-(7), решаются с использованием метода возмущений, путем разложения скорости $V(x, t)$, концентрации носителей заряда $N(x, t)$, эффективной плотности жидкости $M(x, t)$, электрического поля $E(x, t)$, макроскопической энергии $W(x, t)$ и плотности тока $J(x, t)$ по степеням амплитуды действующего электрического поля с сохранением только линейной степени разложения. Интегрирование функции распределения Ферми позволяет в пределе низких температур записать стационарную концентрацию носителей заряда в графене в виде [26]

$$
n=\frac{1}{\pi \hbar^{2}} \frac{V_{\mathrm{F}} E_{\mathrm{F}}^{2}}{\left(V_{\mathrm{F}}^{2}-u^{2}\right)^{3 / 2}},
$$

где $E_{\mathrm{F}}$ и $u-$ соответственно энергия Ферми в отсутствие дрейфа и скорость дрейфа носителей заряда в графене. Затем, используя выражение (8) совместно с линеаризованными уравнениями гидродинамики (1)-(7), получаем выражение для гидродинамической нелокальной комплексной проводимости графена с учетом дрейфа носителей заряда [27]:

$$
\begin{aligned}
& \sigma_{\mathrm{Gr}}(\omega, q)=\frac{i e^{2} n V_{\mathrm{F}} \omega}{E_{\mathrm{F}}\left(\left(1-\beta^{2}\right)(\omega-q u)-3 \beta^{2} i \gamma / 2\right)} \\
& \times \frac{\left(1-\beta^{2}\right)}{q u\left(1-2 \beta^{2}\right) \chi_{1}+\omega^{2}(\omega+i \gamma)\left(1-\beta^{2} / 2\right)+\chi_{1} \chi_{2}-q u \omega \chi_{3}},
\end{aligned}
$$

где $\chi_{1}=q^{2} V_{\mathrm{F}}^{2} / 2, \chi_{2}=\beta^{2}(3 i \gamma+4 \omega)-\omega, \chi_{3}=\beta^{2}(i \gamma-\omega / 2)$ $+(i \gamma 5 / 2+2 \omega), \quad \beta=u / V_{\mathrm{F}}-$,релятивистский“ параметр. Проводимость графена в гидродинамическом режиме без учета постоянного электрического поля была получена в работе [28].
Рассмотрим бесконечный лист однородного графена со смещенным уровнем Ферми, расположенный в плоскости $y=0$. Графен расположен на поверхности подложки $y<0$ с вещественной диэлектрической проницаемостью $\varepsilon_{s}$. Окружающая среда при $y>0$ имеет диэлектрическую проницаемость $\varepsilon_{a}$. Дисперсионное соотношение для плазмонов в данной структуре получено с использованием электродинамического подхода:

$$
\frac{\varepsilon_{a} q_{0}}{\left(q^{2}-q_{0}^{2} \varepsilon_{a}\right)^{1 / 2}}+\frac{\varepsilon_{s} q_{0}}{\left(q^{2}-q_{0}^{2} \varepsilon_{s}\right)^{1 / 2}}=-i \sigma_{\mathrm{Gr}}(\omega, q) Z_{0},
$$

где $Z_{0}=120 \pi-$ волновое сопротивление свободного пространства, $\omega-$ круговая частота, $q-$ волновой вектор плазмона, $q_{0}=\omega / c$. В электростатическом пределе дисперсия (10) совпадает с дисперсией, полученной в [7]. Отклик структуры описывается в виде нелокаль-

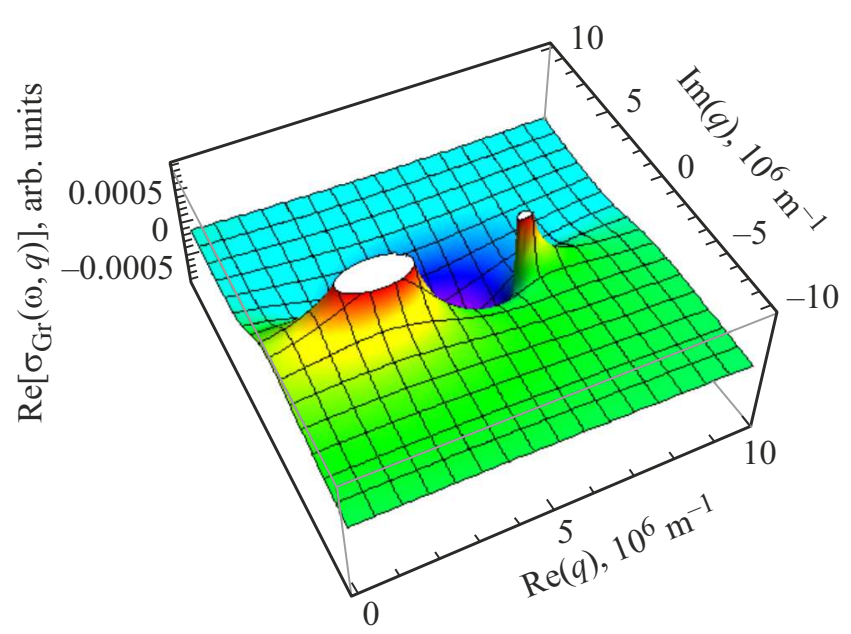

Рис. 1. Действительная часть нелокальной проводимости графена в зависимости от действительной и мнимой частей комплексного волнового вектора плазмона для частоты 0.5 ТГц.

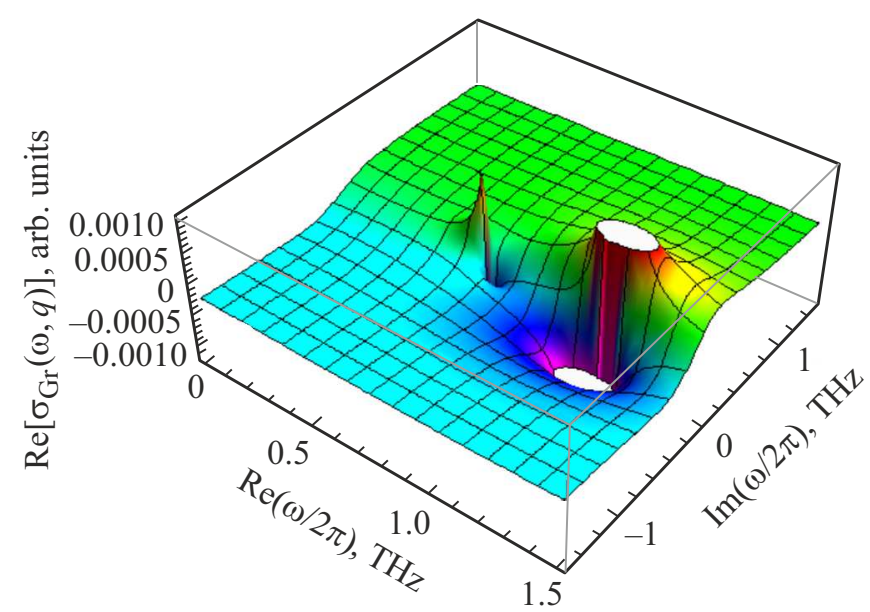

Рис. 2. Действительная часть нелокальной проводимости графена в зависимости от действительной и мнимой частей комплексной частоты плазмона для волнового вектора $q=8 \cdot 10^{6} 1 / \mathrm{M}$ 
ной комплексной динамической проводимости графена $\sigma_{\mathrm{Gr}}(\omega, q)(9)$.

Действительная часть нелокальной комплексной динамической проводимости графена (9) представлена на рис. 1 и 2 соответственно для волноводной и резонаторной постановок задачи. Видно, что действительная часть проводимости (9) может принимать отрицательные значения при некоторых значениях величин комплексных волновых векторов и частот даже при относительно малой величине скорости дрейфа носителей заряда в графене (по сравнению со скоростью Ферми).

Расчеты выполнены для реалистичных параметров графеновых структур при $\tau=1$ пс. Диэлектрические проницаемости окружающей среды и диэлектрической подложки полагались равными $\varepsilon_{a}=\varepsilon_{s}=11.7$ (например $\mathrm{SiC})$. Энергия уровня Ферми допированного графена полагалась равной 75 мэВ, скорость дрейфа носителей заряда в графене полагалась равной $u=0.5 V_{\mathrm{F}}$. Из рис. 1 и 2 наглядно следует неинвариантность волноводного и резонаторного подходов при решении дисперсионного уравнения. Отметим, что решения совпадают только в

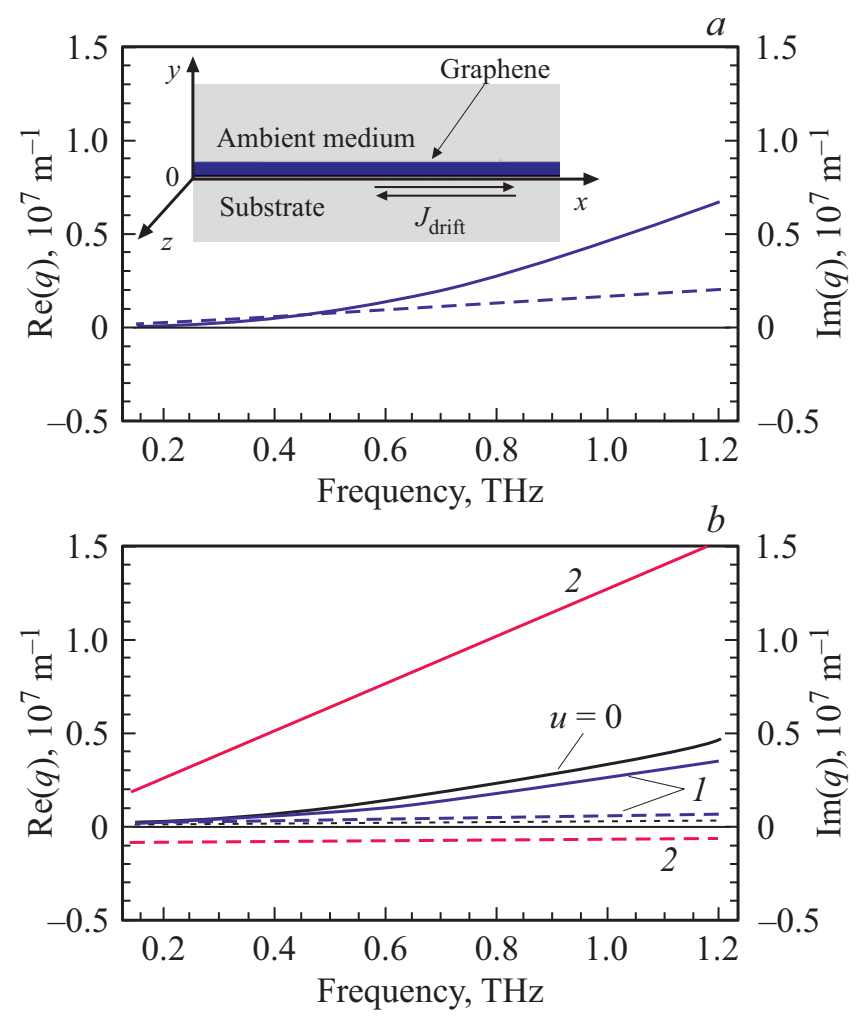

Pис. 3. Действительная (сплошная линия) и мнимая (штриховая линия) части комплексного волнового вектора плазмона в зависимости от действительной ТГц частоты при противонаправленных скорости дрейфа и фазовой скорости плазмона ( $a$, скорость дрейфа носителей заряда $\left.u=-0.5 V_{\mathrm{F}}\right)$ и при сонаправленных скорости дрейфа и фазовой скорости плазмона ( $b$, скорость дрейфа носителей заряда $\left.u=0.5 V_{\mathrm{F}}\right)$. На вставке $(a)$ - схематическое изображение исследуемой структуры и система координат.
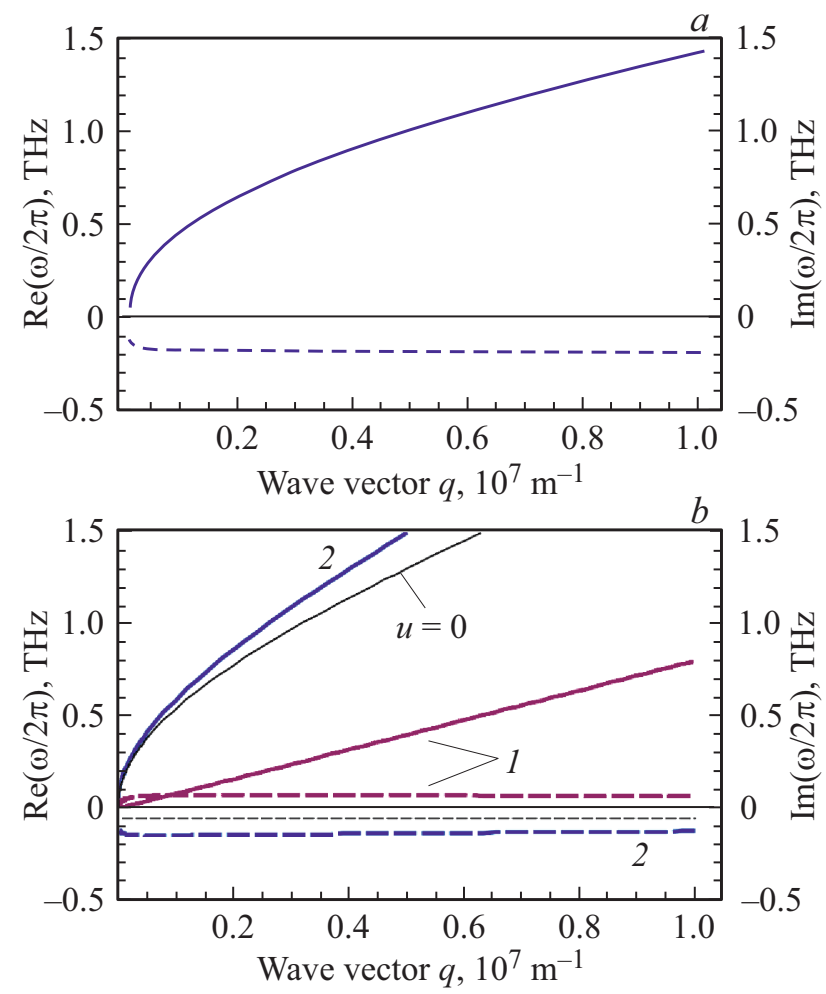

Рис. 4. Действительная (сплошная линия) и мнимая (штриховая линия) части комплексной частоты плазмона в зависимости от действительного волнового вектора плазмона при противонаправленных скорости дрейфа и фазовой скорости плазмона ( $a$, скорость дрейфа носителей заряда $u=-0.5 V_{\mathrm{F}}$ ) и сонаправленных скорости дрейфа и фазовой скорости плазмона $\left(b\right.$, скорость дрейфа носителей заряда $\left.u=0.5 V_{\mathrm{F}}\right)$.

случае отсутствия потерь при действительных значениях частот и волновых векторов.

\section{3. Результаты расчетов и их обсуждение}

Численно найдены значения комплексного волнового вектора плазмона в волноводной постановке задачи в зависимости от действительной ТГц частоты (рис. $3, a, b$ ), и значения комплексной частоты плазмона в резонаторной постановке задачи в зависимости от действительного волнового вектора плазмона (рис. 4, $a, b$ ). Тонкой сплошной линией на рис. $3, b$ и $4, b$ показаны затухающие плазмонные моды при нулевой скорости дрейфа носителей заряда в графене в отсутствие постоянного тока. При ненулевой скорости дрейфа дисперсионная кривая этой плазмонной моды расщепляется на две. Затухающая мода показана кривыми 1 на рис. $3, b$ и кривыми 2 на рис. 4, $b$. Усиливающаяся мода обозначена номером 2 на рис. $3, b$ и номером 1 на рис. $4, b$. Затухание плазмонных мод вызвано преимущественно внутризонными процессами в графене, описываемыми феноменологической скоростью рассеяния импульса но- 
сителей заряда $\tau$. Усиление плазмонных мод происходит по черенковскому механизму, поскольку скорость дрейфа электронов превышает фазовую скорость медленных собственных плазмонных мод во всем рассмотренном частотном диапазоне. Величина затухания/усиления плазмонных возбуждений в графене может варьироваться как за счет изменения скорости рассеяния импульса носителей заряда, так и за счет изменения величины и направления скорости дрейфа носителей заряда в графене. Отметим, что усиливающаяся плазмонная мода существует при любой скорости дрейфа носителей заряда. При этом фазовая скорость усиливающейся моды всегда остается меньше скорости дрейфа. Однако инкремент усиления этой моды монотонно уменьшается до нуля с уменьшением скорости дрейфа. Инкремент достигает существенных величин (>10\% от действительной части волнового вектора моды) при скорости дрейфа больше половины фермиевской скорости.

\section{4. Заключение}

В работе теоретически изучено влияние дрейфа носителей заряда (вследствие приложенного к графену постоянного электрического поля) на плазмонные моды в электронной дираковской жидкости в однородном графене со смещенным уровнем Ферми. Показана возможность усиления плазменных волн по черенковскому механизму в ТГц диапазоне за счет их взаимодействия с дрейфом носителей заряда в графене даже при относительно малой величине скорости дрейфа носителей заряда в графене (по сравнению со скоростью Ферми).

\section{Финансирование работы}

Работа выполнена в рамках государственного задания.

\section{Конфликт интересов}

Авторы заявляют, что у них нет конфликта интересов.

\section{Список литературы}

[1] A.N. Grigorenko, M. Polini, K.S. Novoselov. Nature Photonics, 6, 749 (2012).

[2] A.K. Geim, K.S. Novoselov. Nature Materials, 6, 183 (2007).

[3] M. Orlita, C. Faugeras, P. Plochocka, P. Neugebauer, G. Martinez, D.K. Maude, A.-L. Barra, M. Sprinkle, C. Berger, W.A. de Heer, M. Potemski. Phys. Rev. Lett., 101, 267601 (2008).

[4] T. Otsuji, T. Watanabe, A. El Moutaouakil, H. Karasawa, T. Komori, A. Satou, T. Suemitsu, M. Suemitsu, E. Sano, W. Knap, V. Ryzhii. J. Infr. Millim. Terahertz Waves, 32, 629 (2011).

[5] F.H.L. Koppens, D.E. Chang, F.J. Garcia de Abajo. Nano Lett., 11, 3370 (2011).
[6] V.Ya. Aleskin, A.A. Dubinov, V. Ryzhii. JETP Lett., 89 (2), 63 (2009).

[7] M. Jablan, H. Buljan, M. Soljacic. Phys. Rev. B, 80, 245435 (2009).

[8] O.V. Polischuk, D.V. Fateev, T. Otsuji, V.V. Popov. Appl. Phys. Lett., 111, 081110 (2017).

[9] J.M. Dawlaty, S. Shivaraman, M. Chandrashekhar, F. Rana, M.G. Spencer. Appl. Phys. Lett., 92, 042116 (2008).

[10] I. Gierz, M. Mitrano, J.C. Petersen, C. Cacho, I.C.E. Turcu, E. Springate, A. Stohr, A. Kohler, U. Starke, A. Cavalleri. J. Phys.: Condens. Matter, 27, 164204 (2015).

[11] V. Ryzhii, M. Ryzhii, T. Otsuji. J. Appl. Phys., 101, 083114 (2007).

[12] A. Satou, F.T. Vasko, V. Ryzhii. Phys. Rev. B, 7, 1158431 (2008).

[13] A.A. Dubinov, V.Y. Aleshkin, M. Ryzhii, T. Otsuji, V. Ryzhii. Appl. Phys. Exp., 2, 092301 (2009).

[14] A. Satou, T. Otsuji, V. Ryzhii. Jpn. J. Appl. Phys., 50, 070116 (2011).

[15] A. Satou, V. Ryzhii, Y. Kurita, T. Otsuji. J. Appl. Phys., 113, 143108 (2013).

[16] V. Ryzhii, M. Ryzhii, V. Mitin, T. Otsuji. J. Appl. Phys., 110, 094503 (2011).

[17] A.R. Davoyan, M.Yu. Morozov, V.V. Popov, A. Satou, T. Otsuji. Appl. Phys. Lett., 103, 251102 (2013).

[18] I.V. Oladyshkin, S.B. Bodrov, Yu.A. Sergeev, A.I. Korytin, M.D. Tokman, A.N. Stepanov. Phys. Rev. B, 96, 155401 (2017).

[19] W. Gao, G. Shi, Z. Jin, J. Shu, Q. Zhang, R. Vajtai, P.M. Ajayan, J. Kono, Q. Xu. Nano Lett., 13, 3698 (2013).

[20] D. Svintsov. Phys. Rev. B, 97, 121405(R) (2018).

[21] R. Krishna Kumar, D.A. Bandurin, F.M.D. Pellegrino, Y. Cao, A. Principi, H. Guo, G.H. Auton, M. Ben Shalom, L.A. Ponomarenko, G. Falkovich, K. Watanabe, T. Taniguchi, I.V. Grigorieva, L.S. Levitov, M. Polini, A.K. Geim. Nature Physics, 13, 1182 (2017).

[22] L. Fritz, J. Schmalian, M. Müller, S. Sachdev. Phys. Rev. B, 78, 085416 (2008).

[23] A. Lucas, K. Chung Fong. J. Phys.: Condens. Matter, 30, 053001 (2018).

[24] K. Moors, T.L. Schmidt, O. Kashuba. arXiv:1905.01247v1 [cond-mat.mes-hall] 3 May 2019.

[25] S. Rudin. Int. J. High Speed Electron. Syst., 20 (03), 567 (2011).

[26] D. Svintsov, V. Vyurkov, V. Ryzhii, T. Otsuji. Phys. Rev. B, 88, 245444 (2013).

[27] I.M. Moiseenko, V.V. Popov, D.V. Fateev. AIP Conf. Proc., 2300, 020089 (2020).

[28] D. Svintsov. Phys. Rev. B, 100, 195428 (2019).

Редактор Л.В. Шаронова 


\title{
Features of damping and amplification of terahertz plasmon eigenmodes in graphene with accounting for spatial dispersion
}

\author{
O.V. Polischuk ${ }^{1}$, D.V. Fateev ${ }^{1,2}$, V.V. Popov ${ }^{1}$
}

${ }^{1}$ Kotelnikov Institute

of Radio Engineering and Electronics,

Russian Academy of Sciences, Saratov Branch, 410019 Saratov, Russia

${ }^{2}$ National Research Saratov State University, 410012 Saratov, Russia

\begin{abstract}
In this paper, we consider the effect of charge carrier drift on plasmon modes (plasmons) in electron Dirac liquid in graphene with a shifted Fermi level. Dispersion relations for plasmons were obtained using an electromagnetic approach and a hydrodynamic description of an electron liquid. Damped and amplified plasmon eigenmodes are studied numerically depending on the relationship of the magnitudes and directions of the direct electric current and the phase velocity of the plasmon.
\end{abstract}

